\title{
Exploring the functional connectivity characteristics of brain networks in post-stroke patients with global aphasia: a healthy control based resting-state fMRI study
}

\author{
Xinlei Xu ${ }^{1,2 \#}$, Caili Ren ${ }^{1,3 \#}$, Hui Fang ${ }^{4}$, Zhaohui Li $^{3}$, Ping Chen ${ }^{3}$, Jian Yang ${ }^{5}$, Tong Wang ${ }^{2}$ \\ ${ }^{1}$ Department of Rehabilitation Medicine, The Affiliated Wuxi Mental Health Center of Nanjing Medical University, Wuxi, China; ${ }^{2}$ Department of \\ Rehabilitation Medicine, The First Affiliated Hospital of Nanjing Medical University, Nanjing, China; ${ }^{3}$ Department of Neurorehabilitation, Wuxi \\ Tongren Rehabilitation Hospital, Wuxi, China; ${ }^{4}$ Department of Speech Therapy, Wuxi Tongren Rehabilitation Hospital, Wuxi, China; ${ }^{5}$ Department \\ of Pharmacy, Nanjing Medical University, Nanjing, China \\ Contributions: I) Conception and design: T Wang, C Ren; (II) Administrative support: T Wang, J Yang; (III) Provision of study materials or patients: \\ All authors; (IV) Collection and assembly of data: X Xu, C Ren; (V) Data analysis and interpretation: X Xu, C Ren; (VI) Manuscript writing: All \\ authors; (VII) Final approval of manuscript: All authors. \\ "These authors contributed equally to this work. \\ Correspondence to: Tong Wang. Department of Rehabilitation Medicine, The First Affiliated Hospital of Nanjing Medical University, Nanjing, China. \\ Email: wangtong60621@163.com; Jian Yang. Department of Pharmacy, Nanjing Medical University, Nanjing, China. Email: jianyang@njmu.edu.cn.
}

Background In this study, resting-state functional magnetic resonance imaging (rs-fMRI) was used to investigate the characteristics of functional connectivity of brain networks in patients with post-stroke global aphasia (PGA).

Methods: PGA patients hospitalized in Wuxi Tongren Rehabilitation Hospital during their subacute stage were selected as a case group, and healthy volunteers with matching age, sex, and education level were selected as healthy controls (HCs). rs-fMRI scans were performed to compare the differences of functional connectivity in resting-state networks (RSNs) and in the whole brain between the two groups.

Results: A total of 11 patients with PGA and 11 HCs were included in this study. PGA patients showed decreased inter-hemispheric connectivity of homologs within the sensorimotor network (SMN), salience network, and language network. In the analysis of the whole brain functional connections, PGA patients exhibited both inter-hemispheric and intra-hemispheric hypoconnectivity when compared with HCs. However, they exhibited some stronger connections than HCs between the language network and cerebellar network, which may indicate compensatory mechanisms.

Conclusions: Using rs-fMRI to research differences in the functional connectivity of brain networks in post-stroke global aphasia will help us further understand it's neurological mechanism and provide an important basis for the accurate selection of therapeutic targets in the future to promote better recovery of language function.

Keywords: Aphasia; stroke; resting-state functional magnetic resonance imaging (rs-fMRI); functional connectivity (FC)

Submitted Sep 01, 2021. Accepted for publication Dec 06, 2021.

doi: 10.21037/apm-21-2750

View this article at: https://dx.doi.org/10.21037/apm-21-2750 


\section{Introduction}

Aphasia is a common functional disorder following stroke and affects about one-third of stroke survivors (1). Global aphasia, the most severe form of post-stroke aphasia (PSA), occurs mostly in patients with damage to the middle cerebral artery (MCA) in the dominant hemisphere, which typically leading to infarction or hemorrhage to the left temporo-parieto-frontal perisylvian language areas (2). Due to the serious impairment of language functions, PSA patients may have difficulties in oral expression, listening comprehension, repetition, naming, etc., which will gravely affect their communication abilities and participation in daily activities, resulting in a decline of living quality and a heavy burden on the family and society. Recent years have seen the study of human brain no longer limited to previous morphology, but paid more attention to the combination of morphological and functional information. Blood oxygen level dependent functional magnetic resonance imaging (BOLD-fMRI) technology has been widely used in the research of aphasia, making breakthrough progress. Compared with task-state fMRI, resting-state fMRI (rsfMRI) does not require subjects to perform special tasks and it is especially suitable for patients with severe language problems, and those who are unable to perform task commands.

Biswal (3) and his colleagues were the first to show that the left and right hemispheres of the primary motor network are not dormant during rest, suggesting that information processing and functional connections are taking place between these regions during this time. Greicius et al. (4) found that low-frequency fluctuations of the human brain in the resting state could orderly transmit neural activity information, and different brain regions formed specific functional networks. Therefore, detecting the intrinsic activity of the brain has become an effective way to describe the involvement of cortical functional networks in healthy and clinical populations, and rs-fMRI can reveal anatomically significant and functionally relevant patterns of brain region connections. rs-fMRI studies have shown that the intrinsic activity of relevant brain regions as well as their functional connections may be disrupted after some neurological diseases. Resting-state networks (RSNs) are a group of brain regions that activate with reliable time synchronization when the brain is not actively involved in a particular task, and they can reflect the communications of different regions in the brain. These networks include the default mode network (DMN), executive control network
(ECN), auditory network, visual network, sensorimotor network (SMN), salience network, frontoparietal network (FPN), and dorsal attention network (DAN) $(5,6)$. Gratton et al. (7) investigated the effect of focal lesions on a wide range of brain modular organization in a sample of 35 stroke, traumatic brain injury, and tumor patients, and found that regional focal damages connecting the modules destroyed the modular organization of the brain, even in the undamaged hemisphere. Hillary et al. (8) compared the resting-state networks connections in 22 patients with moderate to severe brain injury during their chronic stage and 15 healthy adults, and found hyperconnectivity patterns in the salience network, ECN, and DMN during rest in patients' group. Geng et al. (9) found reduced functional connectivity (FC) within the DMN, ECN, salience network, auditory network, and cerebellar network in acute brainstem ischemic stroke patients, which was correlated with the measurements of upper limb dysfunction.

Language is an important cognitive function that is supported by a large brain network. Functional imaging indicates (10) that at least one dorsal speech path supports the speech paraphrase and complex grammatical processes, while on the ventral side at least one neural path handles semantic and basic syntactic processes that together form a language network. In recent years, many studies have found that damage to language functional areas may also lead to abnormal metabolic activity in distant undamaged brain areas $(11,12)$. Zhu et al. (13) found reduced connectivity within the left FPN, and between the left FPN and areas of the right frontal cortex in subacute stroke aphasic patients in comparison to controls, which was significantly associated with impaired language comprehension. It is speculated that brain injury may affect comprehension by altering functional connections between regions. In addition to the language function related brain network, other nonlanguage specific related network changes are also involved in the occurrence and development of aphasia $(14,15)$. Durand et al. (16) verified increased resting-state functional connectivity (rsFC) within and between visuo-motor and language areas in patients with chronic moderate to severe aphasia, which probably derived from the integration of visual and sensorimotor systems to sustain certain language tasks.

Brain network connections have shown dynamic changes over the course of stroke $(17,18)$. The subacute stage after stroke is the critical period for language recovery. Most previous fMRI studies on post-stroke aphasia (PSA) did not limit the course of the disease, or specify the type of aphasia. 
Importantly, no study to date has systematically examined the rsFC in subacute global aphasia within RSNs and in the whole brain regions compared with HCs. Thus, we utilized fMRI to study the resting-state functional connectivity characteristics in subacute global aphasia after stroke, expecting to gain a more comprehensive understanding of the neurological mechanism of global aphasia and to explore more effective therapeutic targets for PGA patients.

We present the following article in accordance with the MDAR checklist (available at https://dx.doi.org/10.21037/ apm-21-2750).

\section{Methods}

\section{Subjects}

The case group consisted of 11 patients with global aphasia after ischemic stroke (PGA group), who were hospitalized in Wuxi Tongren Rehabilitation Hospital.

Inclusion criteria: (I) native Chinese speakers aged 40 to 80 years and right-handed; (II) no previous left-sided MCA stroke with a single lesion site verified by CT or MRI; (III) time between 4 and 12 weeks after stroke; (IV) global aphasia was assessed by Western Aphasia Battery (WAB).

Exclusion criteria: (I) visual or hearing impairment affecting language evaluation; (II) unresponsive or unable to cooperate; (III) speech impairment or severe dysarthria before the onset of stroke, or those with other neurodegenerative diseases such as dementia or Parkinson's disease; (IV) history of epilepsy, substance abuse or mental illness; (V) contraindication to MRI scanning (e.g., implanted ferrous metal, claustrophobia).

At the same time, $11 \mathrm{HCs}$ were recruited, meeting the following criteria: age, sex and education level matched with the case group; no major physical diseases, no history of stroke, craniocerebral trauma, neurological, or psychiatric disease; no contraindication to MRI scanning and no abnormal brain structure judged by radiologists.

The PGA group and HCs did not differ for age, sex, or education level. The demographic characteristics for the two groups and the language performance on WAB for PGA group are shown in Table 1.

All procedures performed in this study involving human participants were in accordance with the Declaration of Helsinki (as revised in 2013). The study was approved by the Ethics Committee of Wuxi Tongren Rehabilitation Hospital (No.: WXMHCIRB2015LL009) and informed consent was taken from all the participants. The study protocol was registered in the Chinese Clinical Trial Registry (No.: ChiCTR-IPR-15007382).

\section{Research process}

\section{rs-fMRI data acquisition}

Discovery MR750 3.0T MRI scanner (GE, America) was used to collect the image data of the two groups, and the collected data included the conventional T1-weighted structure images, the whole brain high-resolution structure images, and the resting-state fMRI data. BOLD sensitive functional images were collected using the following parameters: gradient-echo echo-planar imaging pulse sequence, time to repeat (TR) $=2,000 \mathrm{~ms}$, time to echo (TE) $=30 \mathrm{~ms}, 33$ axial slices ( $3.5 \mathrm{~mm}$ thickness), matrix $=64 \times 64$, voxel size $=3.5 \times 3.5 \times 3.5 \mathrm{~mm}^{3}$, flip angle $=90^{\circ}$. Subjects were asked to remain still and awake, and padding was used to minimize head motion.

\section{Image preprocessing}

rs-fMRI data were analyzed using the CONN toolbox for SPM12.

The structural and functional DICOM images obtained from magnetic resonance scanning were converted to NIFTI format. By examining the variation trend of the corresponding data values, we excluded the influence of the unstable signal in the initial stage of MRI scanning, and removed the first 10 images with unstable signal in the transformed data. The rest images were corrected for slice-timing and head movement according to the scanning sequence. Three-step registration approach was used to fit the converted structural NIFTI format data to the images, and then the $\mathrm{T} 1$ structural images were manually redirected. Resegmentation and normalization was implemented to the processed images using the DARTEL template method in the toolbox. The bounding box is [-90,-126,-72;90,90,108], and the voxel size is $3 \times 3 \times 3$. Then the images were smoothed using a 6-mm full-width at half-maximum kernel. Linear trends were removed in the data above and a linear regression of noise sources was performed in the average of the white matter, gray matter, and cerebrospinal fluid (CSF) signals and six head motion parameters. Data were then band-pass filtered, preserving frequencies between 0.01 and $0.08 \mathrm{~Hz}$. After the above preprocessing steps, the data obtained can be statistically analyzed. 
Table 1 Demographic and clinical characteristics for the participants

\begin{tabular}{|c|c|c|c|}
\hline Variable & PGA $(n=11)$ & $\mathrm{HCs}(\mathrm{n}=11)$ & $\mathrm{P}$ \\
\hline Age (years) & $63.82 \pm 12.25$ & $55.91 \pm 7.36$ & $0.081^{b}$ \\
\hline \multicolumn{4}{|l|}{ Education (n) } \\
\hline Primary school & 3 & 4 & $0.478^{\circ}$ \\
\hline Senior high school & 2 & 1 & \\
\hline College degree or above & 2 & 1 & \\
\hline Time post stroke (days) & $49.00 \pm 19.91$ & & \\
\hline Aphasia quotient & $14.53 \pm 11.91$ & & \\
\hline Repetition score & $2.12 \pm 1.91$ & & \\
\hline Naming score & $1.59 \pm 2.50$ & & \\
\hline
\end{tabular}

${ }^{\mathrm{a}}$, Chi-square test; ${ }^{\mathrm{b}}$, independent samples $t$-test; ${ }^{\mathrm{c}}$, nonparametric test.

\section{ROI selection and statistical analysis}

Firstly, the rsFC of PGA and HCs in 8 RSNs was analyzed. These regions are result of brain region segmentation and extraction obtained from the independent component analysis (ICA) algorithm based on the rs-fMRI data of 497 healthy volunteers in CONN toolbox provided by the human connectome project (HCP) (19). The purpose was to define regions of interest (ROIs) in different functional networks, which were then divided into 8 sub-networks and 32 ROIs. The 8 sub-networks and 32 ROIs were DMN [medial prefrontal cortex (MPFC), left/right lateral parietal cortex (LP), posterior cingulate cortex (PCC)], SMN [left/ right lateral sensorimotor cortex (lateral), superior lateral sensorimotor cortex (superior)], visual network [medial visual cortex (medial), occipital visual cortex (occipital), left/right lateral visual cortex (lateral)], salience network [anterior cingulate cortex (ACC), left/right anterior insula (AInsula), left/right rostral prefrontal cortex (RPFC), left/right supramarginal gyrus (SMG)], DAN [left/right frontal eye field (FEF), left/right intraparietal sulcus (IPS)], FPN [left/right lateral prefrontal cortex (LPFC), left/ right posterior parietal cortex (PPC)], language network [left/right inferior frontal gyrus (IFG), left/right posterior superior temporal gyrus (pSTG)], cerebellar network [anterior cerebellum (anterior), posterior cerebellum (posterior)]
Subsequently, rsFC analysis was performed in the whole brain. fMRI data were divided into 132 cortical, subcortical, and cerebellar ROIs. Cortical $(\mathrm{n}=91)$ and subcortical ( $\mathrm{n}=15)$ ROIs were from the FSL Harvard-oxford atlas HarvardOxford-cort-maxprob-thr25-1 mm.nii and HarvardOxford-sub-maxprob-thr25-1 mm.nii), respectively. The cerebellar portion $(n=26)$ was derived from automated anatomical labeling (AAL) (20).

ROI correlation analysis was carried out by the functional connectivity toolbox (Conn version 18a; http:// www.nitrc.org/projects/conn). The entire matrix of ROIto-ROI functional connectivity values were computed for each subject using bivariate correlation measure. The effect of functional connectivity was corrected for multiple comparisons using the false discovery rate (FDR) approach with a significance threshold of $\mathrm{P}<0.05$.

\section{Results}

\section{Differences of rsFC in RSNs between the case group and} the control group

As shown in Figure 1, FC analysis was performed based on 32 ROIs of the above 8 RSNs. These preliminary results showed that PGA patients exhibited decreased interhemispheric rsFC of homologs within the SMN (lateral sensorimotor cortex), salience network [supramarginal 


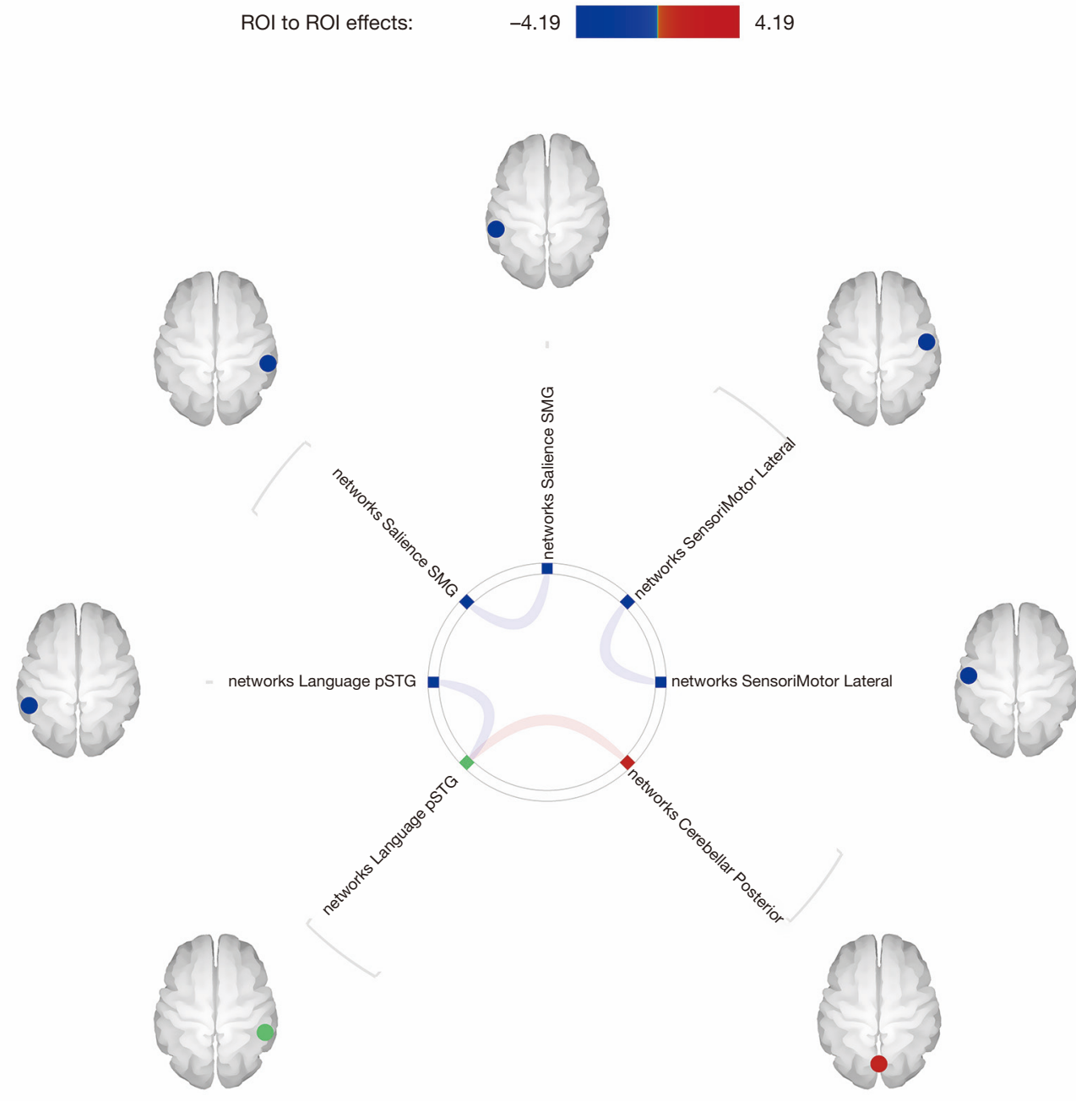

Figure 1 The differences in functional connections within resting-state networks for PGA patients compared with HCs. The left and right hemisphere directions consistent with the figure. Red/blue lines represent enhanced/reduced functional connectivity. SMG, supramarginal gyrus; lateral, lateral sensorimotor cortex; posterior, posterior cerebellum; pSTG, posterior superior temporal gyrus.

gyrus (SMG)], and language network [posterior superior temporal gyrus (pSTG)] compared with HCs. However, PGA patients showed some stronger connections than HCs between the language network (right pSTG) and cerebellar network (posterior cerebellum). The statistical data of the different rsFC in PGA compared with HCs is shown in Table 2.

\section{Differences of rsFC in the whole brain network between the two groups based on the Harvard-Oxford atlas and AAL}

In comparison to HCs, PGA group exhibited an overall pattern of reduced connectivity both inter-hemispheric and intra-hemispheric (see Figure 2). Figure 3 showed 3D rendering of reduced functional connections of the whole brain. The statistical data of the different rsFC in PGA compared with HCs is shown in Table 3.

\section{Differences of rsFC between bilateral hemispheres based on the Harvard-Oxford atlas and $A A L$}

Figure 4 shows a wide range of decreased FC between the bilateral hemispheres, especially between regions in bilateral temporal lobes [planum polare (PP), Heschl's gyrus 
Table 2 Statistical data of different functional connections within resting-state networks for PGA patients compared with HCs

\begin{tabular}{lccc}
\hline Seed regions & Connected regions & T & P-unc \\
\hline Sensorimotor network. Lateral(L) & Sensorimotor network. Lateral(R) & -3.91 & 0.0009 \\
Salience networks. SMG(L) & Salience networks. SMG(R) & -4.19 & 0.0005 \\
Language networks. pSTG(R) & Cerebellar networks. Posterior & 3.50 & 0.0022 \\
& Language networks. pSTG(L) & -3.42 & 0.0027 \\
\hline
\end{tabular}

P-unc, uncorrected P value; P-FDR, P value corrected by FDR; Lateral, lateral sensorimotor cortex; SMG, supramarginal gyrus; pSTG, posterior superior temporal gyrus; Posterior, posterior cerebellum.

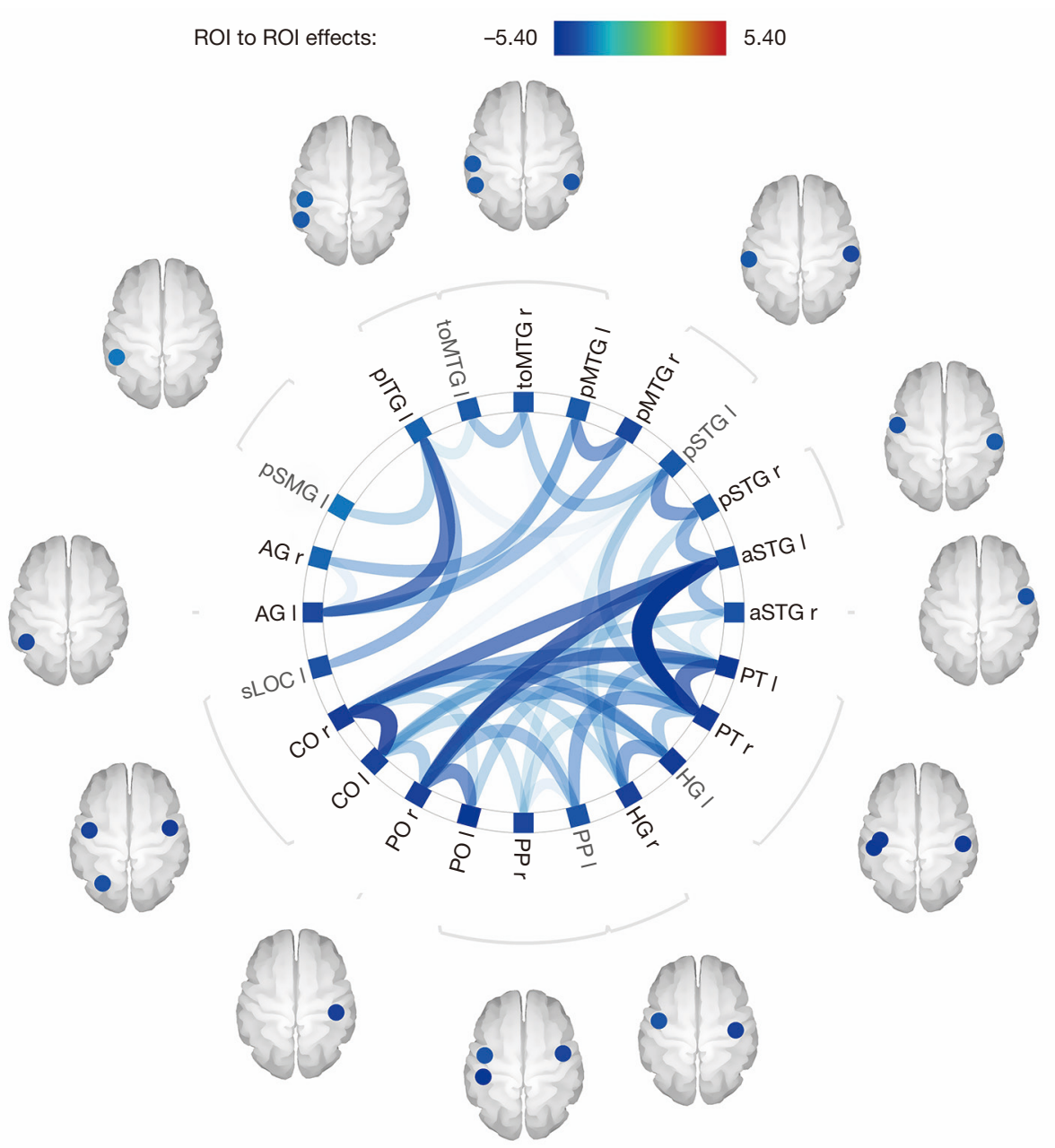

Figure 2 The differences in the whole brain functional connections for PGA patients compared with HCs. The left and right hemisphere directions consistent with the figure. Blue lines represent reduced functional connectivity. 1, left hemisphere; r, right hemisphere; AG, angular gyrus; sLOC, superior lateral occipital cortex; CO, central operculum; PO, parietal operculum; PP, planum polare; HG, Heschl's gyrus; PT, planum temporale; aSTG, anterior superior temporal gyrus; pSTG, posterior superior temporal gyrus; pMTG, posterior middle temporal gyrus; toMTG, temporooccipital part of middle temporal gyrus; pITG, posterior inferior temporal gyrus; pSMG, posterior supramarginal gyrus. 

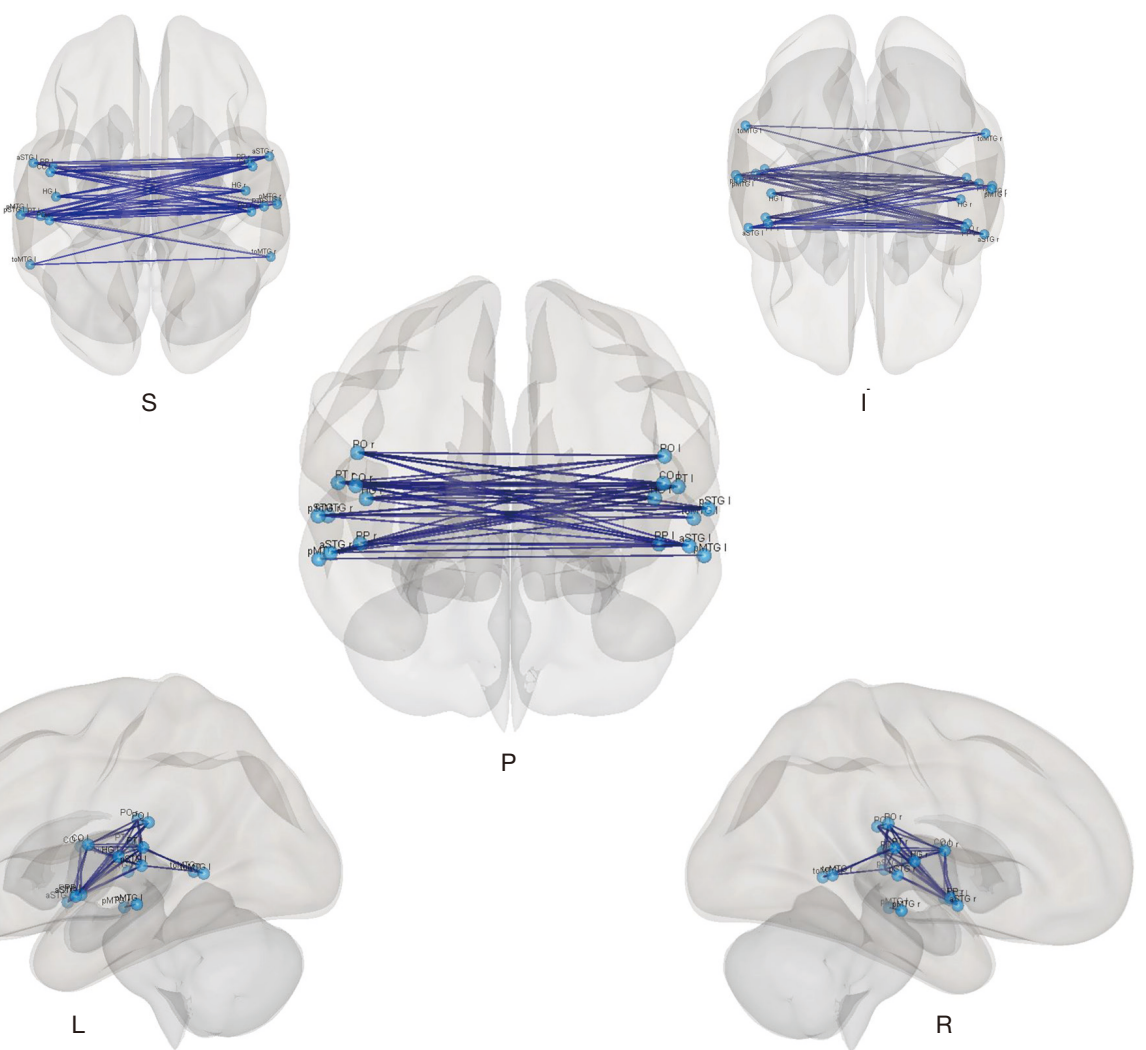

Figure 3 3D rendering of the differences in the whole brain functional connections for PGA patients compared with HCs. S, superior; I, inferior; P, posterior; L, left; R, right.

(HG), planum temporale (PT), anterior superior temporal gyrus (aSTG), posterior superior temporal gyrus (pSTG), posterior middle temporal gyrus (pMTG), temporooccipital part of middle temporal gyrus (toMTG)], bilateral central operculum (CO) and parietal operculum (PO).

\section{Differences of rsFC within the unilateral hemisphere based on the Harvard-Oxford atlas and AAL}

As shown in Figure 5, the analysis of intra-hemispheric rsFC revealed significantly reduced FC between the left posterior inferior temporal gyrus (pITG) and angular gyrus (AG), superior lateral occipital cortex (sLOC) and posterior supramarginal gyrus (pSMG) in PGA compared with HCs.

\section{Discussion}

As a complex neurofunctional organ, the brain often accomplishes specific tasks through the coordination and cooperation of anatomically discrete brain regions. During a stroke, the blood perfusion reduces suddenly in the brain areas providied by the affected blood vessels. In addition to the corresponding cerebral cortex and subcortical tissues which are structurally and functionally damaged, the perilesional and remote areas will also be affected (21). rs-fMRI shows changes in activity of different brain areas with high spatial-temporal resolution. By measuring the temporal correlation of BOLD signals in different areas of the brain, we can identify RSNs and study the functional connectivity properties. Previous studies have confirmed the existence of a synchronization of electrophysiological signals in the RSNs, and the changes of FC are related to the clinical manifestations of disease under pathological status.

Language is an advanced neurological function realized in numerous brain networks, and aphasia can be caused by direct focal damage to eloquent cortical or subcortical areas as well as by dysfunction of interconnected remote areas related to the language network $(12,13)$. Rehabilitation of aphasia is based on the theory of neuroplasticity. Apart 
Table 3 Statistical data of different functional connections in the whole brain for PGA patients compared with HCs

\begin{tabular}{|c|c|c|c|c|}
\hline Seed regions & Connected regions & $\mathrm{T}$ & P-unc & P-FDR \\
\hline \multirow{4}{*}{ PO r } & PT I & -4.17 & 0.0005 & 0.0042 \\
\hline & POI & -4.10 & 0.0006 & 0.0042 \\
\hline & HG I & -3.62 & 0.0017 & 0.0079 \\
\hline & $\mathrm{COI}$ & -3.43 & 0.0026 & 0.0101 \\
\hline \multirow[t]{6}{*}{ PT $r$} & aSTG I & -5.40 & 0.0000 & 0.0006 \\
\hline & PT I & -4.37 & 0.0003 & 0.0034 \\
\hline & PP I & -3.80 & 0.0011 & 0.0086 \\
\hline & pSTG I & -3.36 & 0.0031 & 0.0107 \\
\hline & POI & -3.34 & 0.0032 & 0.0107 \\
\hline & toMTG I & -2.72 & 0.0133 & 0.0383 \\
\hline \multirow[t]{5}{*}{$\mathrm{COr}$} & $\mathrm{COI}$ & -4.72 & 0.0001 & 0.0030 \\
\hline & aSTG I & -4.30 & 0.0003 & 0.0040 \\
\hline & HG I & -3.82 & 0.0011 & 0.0074 \\
\hline & PT I & -3.74 & 0.0013 & 0.0074 \\
\hline & POI & -3.17 & 0.0049 & 0.0210 \\
\hline PP r & PP I & -2.95 & 0.0079 & 0.0452 \\
\hline \multirow[t]{2}{*}{ toMTG r } & toMTG I & -3.51 & 0.0022 & 0.0276 \\
\hline & pSTG I & -3.47 & 0.0024 & 0.0276 \\
\hline \multirow[t]{2}{*}{ pMTG r } & pMTG I & -3.98 & 0.0007 & 0.0169 \\
\hline & AG I & -3.66 & 0.0016 & 0.0178 \\
\hline \multirow[t]{3}{*}{ pSTG $r$} & pSTG I & -4.00 & 0.0007 & 0.0156 \\
\hline & aSTG I & -3.72 & 0.0014 & 0.0156 \\
\hline & PT I & -3.18 & 0.0047 & 0.0358 \\
\hline$A G r$ & pMTG I & -3.62 & 0.0017 & 0.0392 \\
\hline
\end{tabular}

Table 3 (continued) 
Table 3 (continued)

\begin{tabular}{|c|c|c|c|c|}
\hline Seed regions & Connected regions & $\mathrm{T}$ & P-unc & P-FDR \\
\hline \multirow{3}{*}{$\mathrm{HG} \mathrm{r}$} & PT I & -3.62 & 0.0017 & 0.0198 \\
\hline & $\mathrm{COI}$ & -3.25 & 0.0040 & 0.0236 \\
\hline & aSTG I & -3.24 & 0.0041 & 0.0236 \\
\hline \multirow[t]{2}{*}{ aSTG $r$} & aSTG I & -3.63 & 0.0017 & 0.0368 \\
\hline & COI & -3.35 & 0.0032 & 0.0368 \\
\hline \multirow[t]{2}{*}{ PT I } & aSTG $r$ & -2.98 & 0.0074 & 0.0283 \\
\hline & toMTG r & -2.78 & 0.0114 & 0.0376 \\
\hline \multirow[t]{2}{*}{ AG I } & $A G r$ & -2.93 & 0.0083 & 0.0478 \\
\hline & pMTG I & -3.16 & 0.0050 & 0.0382 \\
\hline \multirow[t]{5}{*}{ plTG l } & AG I & -4.31 & 0.0003 & 0.0078 \\
\hline & sLOC I & -3.76 & 0.0012 & 0.0142 \\
\hline & pSMG I & -3.35 & 0.0032 & 0.0247 \\
\hline & toMTG I & -3.04 & 0.0065 & 0.0373 \\
\hline & pSTG I & -2.87 & 0.0095 & 0.0435 \\
\hline
\end{tabular}

P-unc, uncorrected P value; P-FDR, P value corrected by FDR; I, left; r, right; PO, parietal operculum; PT, planum temporale; CO, central operculum; PP, planum polare; toMTG, temporooccipital part of middle temporal gyrus; pMTG, posterior middle temporal gyrus; pSTG, posterior superior temporal gyrus; AG, angular gyrus; HG, Heschl's gyrus; aSTG, anterior superior temporal gyrus; pITG, posterior inferior temporal gyrus; sLOC, superior lateral occipital cortex; pSMG, posterior supramarginal gyrus.

from the natural recovery of language function, speech and language therapy (SLT) plays an important role in the recovery of aphasia, although it is less effective in PGA patients due to their seriously impaired auditory comprehension. Non-invasive brain stimulation (NIBS), including transcranial magnetic stimulation (TMS) and transcranial direct current stimulation (tDCS), can regulate the activity of the cerebral cortex, and thus has been widely used in aphasic rehabilitation research. The therapeutic effects of NIBS are influenced by the intensity, frequency and target site of the stimulation. Although the impact of focal lesions on language function in PSA has been widely researched (22-24), the injury mechanism of language network and its influence on other functional connections in the brain remains unclear. Exploring the functional connectivity of brain networks in global aphasia is critical for further understanding the abnormal language performance and revealing its injury and recover mechanisms. Sandberg et al. (14) compared the connections of RSNs of 7 patients with chronic aphasia and $11 \mathrm{HCs}$, and demonstrated decreased FC both inter-hemisphere and intra-hemisphere in the DMN, ECN, SMN, auditory network and salience network in patient group. The results were similar to those of ours. In this study, we used rs-fMRI to explore the functional connectivity of brain networks in 11 subacute stroke patients with global aphasia and 11 healthy adults, and found that compared with HCs, PGA patients presented hypoconnectivity both between bilateral hemispheres and within the left hemisphere.

In our study, PGA group showed significantly decreased rsFC than HCs between bilateral SMG of the salience network as well as both lateral cortex of the SMN. Several previous studies have confirmed a pattern of hypoconnectivity in RSNs of aphasia patients, which may be 


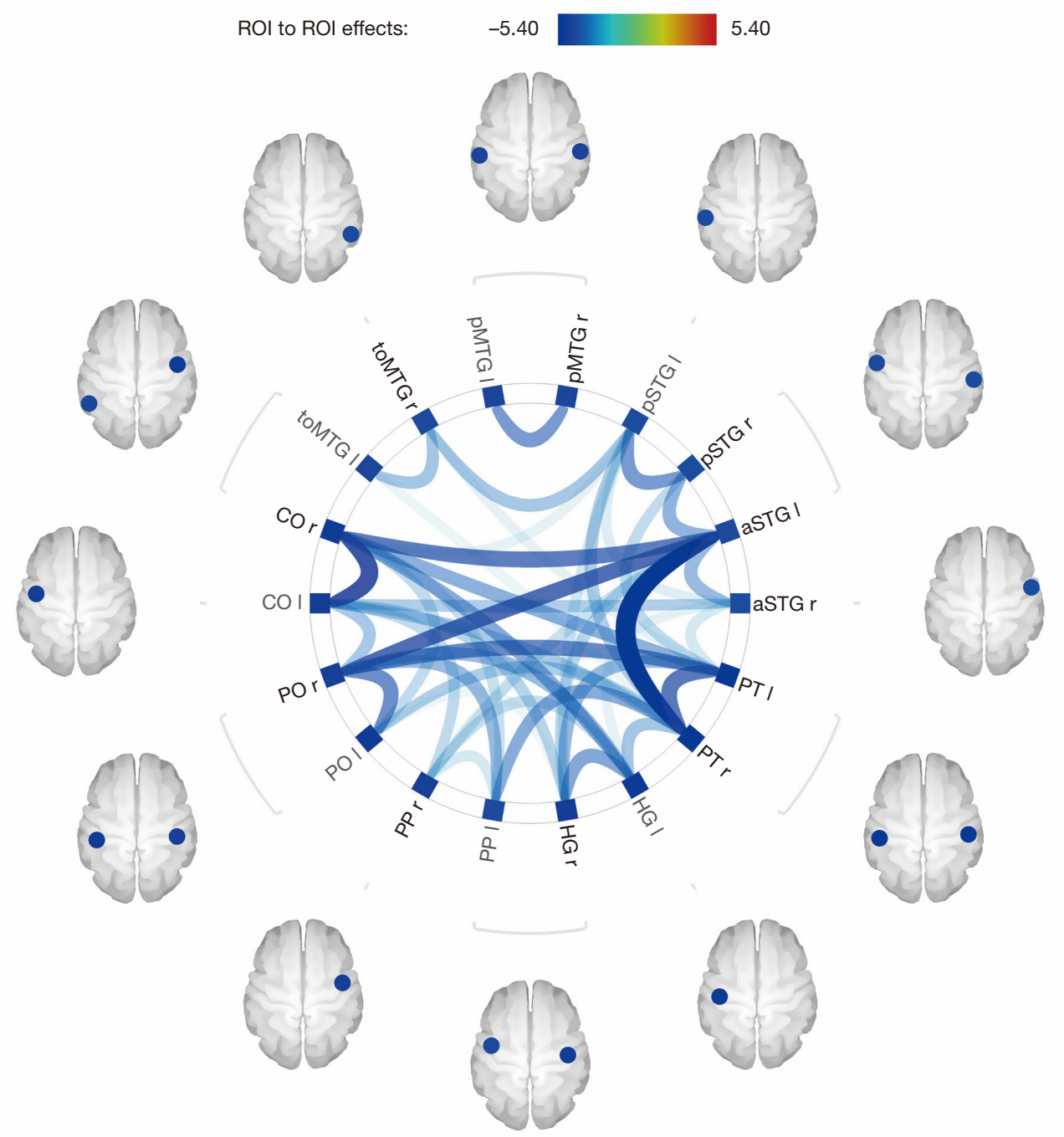

Figure 4 The differences of inter-hemispheric functional connections for PGA patients compared with HCs. The left and right hemisphere directions consistent with the figure. Blue lines represent reduced functional connectivity. 1, left hemisphere; r, right hemisphere; CO, central operculum; PO, parietal operculum; PP, planum polare; HG, Heschl's gyrus; PT, planum temporale; aSTG, anterior superior temporal gyrus; pSTG, posterior superior temporal gyrus; pMTG, posterior middle temporal gyrus; toMTG, temporooccipital part of middle temporal gyrus.

associated with the severity of aphasia $(13,14,25,26)$. Naeser et al. (27) demonstrated that the improvement of naming in chronic aphasia was related to the enhancement of rsFC in the DMN and SN. The SMG of dominant hemisphere is thought to be responsible for repetition and damage to this region may lead to conduction aphasia. The SMG is also involved in planning and execution of speech articulation (28), and lesions in the SMG and AG are associated with poor recovery of naming (29). The SMN supports auditory feedback processing during language expression (30), so patients with injury in SMN may also present with repetition disorder (31). In addition, we found decreased inter-hemispheric rsFC in pSTG of the language network, which was later confirmed by the whole brain network connectivity analysis based on the Harvard-Oxford atlas. The left inferior frontal gyrus (IFG) and the pSTG are key areas of language network, representing motor and sensory language center respectively. In comparison to the frontal lobe, pSTG plays a more critical role in complex syntactic processing (32). A positron emission tomography (PET) 

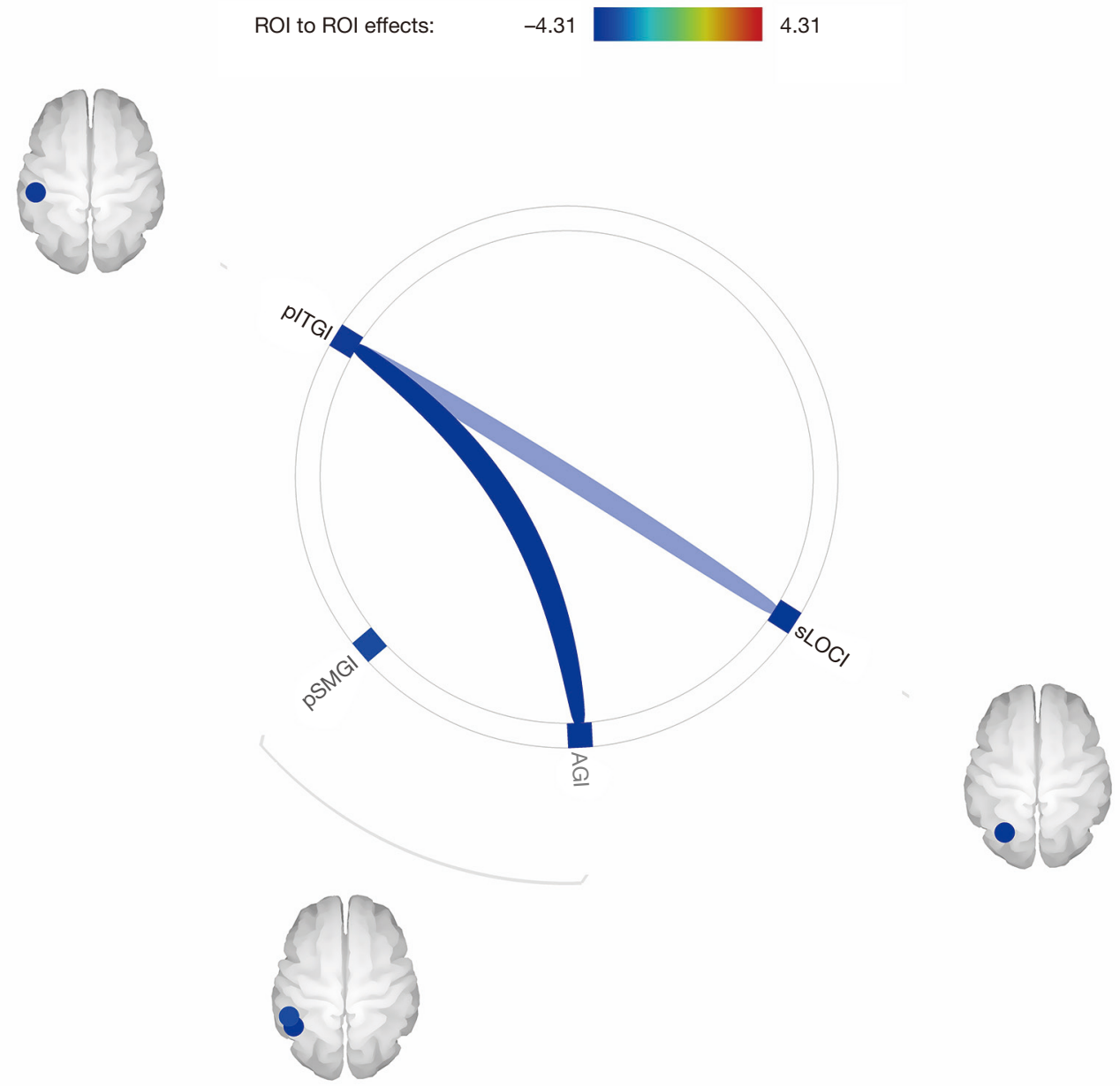

Figure 5 The differences of intra-hemispheric functional connections for PGA patients compared with HCs. The left and right hemisphere directions consistent with the figure. Blue lines represent reduced functional connectivity. 1, left hemisphere; pITG, posterior inferior temporal gyrus; pSMG, posterior supramarginal gyrus; AG, angular gyrus; sLOC, superior lateral occipital cortex.

based study (33) confirmed that the FC between bilateral temporal lobes was decreased in post-stroke aphasia patients compared with healthy controls, and the degree of language dysfunctions was correlated with the severity of injury, suggesting that reduced FC between bilateral hemispheres, especially between the language areas and their homologs may be one of the neural mechanisms of aphasia after stroke.

Our study revealed that PGA exhibited stronger rsFC than HCs between the posterior cerebellum of the cerebellar network and the right pSTG of the language network in PGA group, which may indicate a compensory mechanism. The right pSTG is the homolog of the Wernicke's area and numerous studies have shown that lesions in the left hemisphere can lead to the selective participation of the right hemisphere in language function (34), possibly due to disinhibition of the right hemisphere in the presence of left hemisphere lesions. There is plenty of evidence showing that inhibitory repetitive transcranial magnetic stimulation (rTMS) over the unafected hemisphere can improve language function in PSA patients with left hemispheric lesions $(35,36)$. Ren et al. (37) conducted a randomized sham-controlled study and demonstrated that inhibitory rTMS targeting the right pSTG can signifcantly improve language recovery in terms of auditory comprehension and repetition, providing a new option for NIBS targets on language recovery. The cerebellum communicates with extensive areas of the cerebral cortex including language functional regions via polysynaptic circuits (38), and plays an important role in cognitive, emotional, behavioral and 
language functions $(39,40)$. A meta-analysis (41) clarified that patients with isolated cerebellar lesions had severe deficits in language function, which was manifested in reading, naming and verbal fluency. The cerebellum is involved in all kinds of language processing, such as speech production, word retrieval, semantic processing, working memory, and language learning (42), and even plays an important role in the multilingual control network (43). Sebastian et al. (44) reported for the first time that using tDCS to stimulate the right cerebellum in a patient with chronic aphasia after bilateral hemispheric stroke. Significant improvements in spelling and writing speed were found in patients treated with anodic tDCS, and enhanced FC was confirmed by rs-fMRI between undamaged areas of the right cerebellum and bilateral cerebral hemispheres after treatment, providing a new idea for the target selection of NIBS in the treatment of PSA. All PGA patients included in this study had infarction in MCA territory, of which the cortical and subcortical structures were severely damaged, and the compensatory capacity of peripheral tissue for functional reorganization was limited. Therefore, the enhancement of rsFC between the posterior cerebellum and the right pSTG may reflect neuroplasticity mechanism for subacute PGA patients. These findings provide further understanding of local lesion effects on the cortical network as a whole, which can guide the selection of therapeutic targets for PGA and promote neural reorganization for optimal functional recovery.

In the analysis of $\mathrm{rsFC}$ in the whole brian, we found that compared with HCs, PGA exhibited extensively reduced FC between bilateral hemispheres, including multiple ROIs in the temporal lobe, central operculum (CO) and parietal operculum (PO). Lesions in the left cerebral hemisphere can lead to abnormal functional activity in the contralateral cerebral hemisphere and disrupt the balance between the two sides of the brain in the resting state. The temporal lobe has always been considered as an important region for semantic processing and sentence production, and comprehension defificits were associated with lesions in the temporal lobe, including the anterior and posterior superior temporal gyri (aSTG, pSTG), the anterior and posterior middle temporal gyri (aMTG, pMTG), the auditory association areas [planum temporale (PT) and planum polare (PP)], the temporal pole (TP), the MTG temporooccipital (toMTG) regions, superior marginal gyrus (SMG), angular gyrus (AG), CO and PO. Li et al. (45) found that the regional neuronal activity in left pMTG was correlated with the severity of chronic aphasia. Sebastian and his colleagues (46) observed four aphasia patients due to posterior cerebral artery stroke and found that improved naming skills were associated with increased $\mathrm{FC}$ within and between the left and right hemispheres language regions (IFG, STG, MTG, inferior temporal gyrus (ITG), SMG and $A G)$, while the patients of whom the language function did not improve showed an opposite pattern. The extensive reduction of inter-hemispheric $\mathrm{rsFC}$ in our study may explain the manifestation of severe impairment of language function and the decreased FC between the language functional areas and their homologous regions may be one of the injury mechanisms of PGA. Xu et al. (47) found that in patients with non-fluent aphasia due to basal ganglia region infarction, the improvement of language fluency was related to the enhancement of FC in bilateral Broca's and Wernicke's areas, suggesting that increased FC across hemispheres may be a compensatory mechanism for the improvement of subcortical aphasia symptoms.

In the analysis of intra-hemispheric connections, significantly decreased rsFC between the left posterior inferior temporal gyrus (pITG) and the AG has been certified in PGA group compared with HCs. In addition, the differences of rsFC between pITG and sLOC, pSMG were also statistically significant. The left ITG in which the visual memory was storage serves an important role in language comprehension and oral fluency (48), thus lesions in this area may result in dyslexia (49). Lin et al. (50) identified five major connections of ITG connecting to other gyri in the temporal, frontal, and parietal lobes: the arcuate fasciculus (AF), inferior longitudinal fasciculus (ILF), U-fiber, uncinate fasciculus (UF), and vertical occipital fasciculus (VOF). The VOF, with connections to the angular gyrus, fusiform gyrus, and occipital lobe, is critical for highorder functions, such as facial recognition, reading, and concept formation. The inferior parietal lobule, composed of the AG and SMG, is located at the junction of temporal, parietal, and occipital lobes, and is also the intersection and overlapping area of sight, hearing, and touch, and has been proved to be associated with auditory comprehension, lexical repetition and semantic processing (51). The auditory information is first transmitted to the temporalparietal-occipital association cortex, of which the AG is the center, and then transmitted to the Wernicke's area. Ramage et al. (52) demonstrated that connections between the left AG and left ITG was the only significant predictor of auditory comprehension scores in chronic aphasia patients. The occipital lobe is thought to be mainly related to visual information integration. Mårtensson et al. (53) 
observed sensory-specific anomic aphasia patients following left occipital lesions, and found that these patients featured with different levels of semantic specificity as well as visionrelated sensory information lexical difficulty, which may due to difficult obtaintion of visual semantic information. Reduced rsFC within the left hemisphere in PGA may reflect the decline of compensatory ability in language functional areas, which may indicate a disconnection reaction result from inhibition of the contralateral hemisphere through the corpus callosum.

Therefore, in summary, the study of resting-state functional connectivity in subacute PGA not only exhibited local injury to the whole brain network, but also reflected the reorganization mechanism of the brain. There are a few limitations of this study, mainly due to the small sample size of each group. Moreover, we didn't analyse the correlation between language proficiency score and resting-state functional connectivity, on account of overall lower scores in PGA patients.

\section{Conclusions}

Brain network functional connectivity analysis based on rs-fMRI plays a positive effect on the research of injury and compensatory mechanism of subacute global aphasia, and divert our attention from unilateral effects of injury to bilateral hemispheres. However, how brain regions influence each other and the dynamic changes of brain functional connections in different courses of the disease worth further investigation. In this study, we found that subacute PGA patients showed decreased inter-hemispheric rsFC within the sensorimotor network, salience network, and language network. From the perspective of whole brain network connectivity, the rsFC between bilateral temporal lobes and between the pITG and AG within the left hemisphere decreased most significantly. However, PGA presented enhanced connectivity between the language network and cerebellar network, suggesting a compensatory mechanism.

Using rs-fMRI to study the functional connectivity characteristics of aphasia lays a foundation for revelation of its injury and compensatory mechanisms and provides a basis for the accurate selection of therapeutic targets to promote better neural reorganization, and the evaluation of different therapeutic effect. Future work will explore the neurological mechanisms of post-stroke aphasia and the effect of different treatments on rs-fMRI network connectivity in a larger population.

\section{Acknowledgments}

The authors thank Dr. Shutian Xu at Shanghai University of Traditional Chinese Medicine for his help with fMRI data analyses.

Funding: This study was funded by grants from the National Natural Science Foundation of China (No. 81501949), Natural Science Foundation of Jiangsu Province (No. BK20201138), Wuxi Taihu Talent Project (No. WXTTP2020008), Top Talent Support Program for Young and Middle-aged People of Wuxi Health Committee and General Project from Wuxi Health Commission (No. MS201911).

\section{Footnote}

Reporting Checklist: The authors have completed the MDAR checklist. Available at https://dx.doi.org/10.21037/ apm-21-2750

Data Sharing Statement: Available at https://dx.doi. org/10.21037/apm-21-2750

Conflicts of Interest: All authors have completed the ICMJE uniform disclosure form (available at https://dx.doi. org/10.21037/apm-21-2750). The authors have no conflicts of interest to declare.

Ethical Statement: The authors are accountable for all aspects of the work in ensuring that questions related to the accuracy or integrity of any part of the work are appropriately investigated and resolved. All procedures performed in this study involving human participants were in accordance with the Declaration of Helsinki (as revised in 2013). The study was approved by the Ethics Committee of Wuxi Tongren Rehabilitation Hospital. (No.: WXMHCIRB2015LL009) and informed consent was taken from all the participants. The study protocol was registered in the Chinese Clinical Trial Registry (No.: ChiCTRIPR-15007382).

Open Access Statement: This is an Open Access article distributed in accordance with the Creative Commons Attribution-NonCommercial-NoDerivs 4.0 International License (CC BY-NC-ND 4.0), which permits the noncommercial replication and distribution of the article with the strict proviso that no changes or edits are made and the original work is properly cited (including links to both the 
formal publication through the relevant DOI and the license). See: https://creativecommons.org/licenses/by-nc-nd/4.0/.

\section{References}

1. Correction to: Heart Disease and Stroke Statistics-2017 Update: A Report From the American Heart Association. Circulation 2017;136:e196.

2. Kiran $\mathrm{S}$. What is the nature of poststroke language recovery and reorganization? ISRN Neurol 2012;2012:786872.

3. Biswal B, Yetkin FZ, Haughton VM, et al. Functional connectivity in the motor cortex of resting human brain using echo-planar MRI. Magn Reson Med 1995;34:537-41.

4. Greicius MD, Krasnow B, Reiss AL, et al. Functional connectivity in the resting brain: a network analysis of the default mode hypothesis. Proc Natl Acad Sci U S A 2003;100:253-8.

5. Raichle ME. The restless brain. Brain Connect 2011;1:3-12.

6. Fedorenko E, Duncan J, Kanwisher N. Broad domain generality in focal regions of frontal and parietal cortex. Proc Natl Acad Sci U S A 2013;110:16616-21.

7. Gratton C, Nomura EM, Pérez F, et al. Focal brain lesions to critical locations cause widespread disruption of the modular organization of the brain. J Cogn Neurosci 2012;24:1275-85.

8. Hillary FG, Rajtmajer SM, Roman CA, et al. The rich get richer: brain injury elicits hyperconnectivity in core subnetworks. PLoS One 2014;9:e104021.

9. Geng W, Zhang J, Shang S, et al. Reduced functional network connectivity is associated with upper limb dysfunction in acute ischemic brainstem stroke. Brain Imaging Behav 2021. [Epub ahead of print].

10. Friederici AD, Gierhan SM. The language network. Curr Opin Neurobiol 2013;23:250-4.

11. Tippett DC. Update in Aphasia Research. Curr Neurol Neurosci Rep 2015;15:49.

12. Siegel JS, Ramsey LE, Snyder AZ, et al. Disruptions of network connectivity predict impairment in multiple behavioral domains after stroke. Proc Natl Acad Sci U S A 2016;113:E4367-76.

13. Zhu D, Chang J, Freeman S, et al. Changes of functional connectivity in the left frontoparietal network following aphasic stroke. Front Behav Neurosci 2014;8:167.

14. Sandberg CW. Hypoconnectivity of Resting-State
Networks in Persons with Aphasia Compared with Healthy Age-Matched Adults. Front Hum Neurosci 2017;11:91.

15. Wang $X$, Wang $M$, Wang W, et al. Resting state brain default network in patients with motor aphasia resulting from cerebral infarction. Chinese Science Bulletin 2014;59:4069-76.

16. Durand E, Masson-Trottier M, Sontheimer A, et al. Increased links between language and motor areas: A proof-of-concept study on resting-state functional connectivity following Personalized Observation, Execution and Mental imagery therapy in chronic aphasia. Brain Cogn 2021;148:105659.

17. Hu J, Du J, Xu Q, et al. Dynamic Network Analysis Reveals Altered Temporal Variability in Brain Regions after Stroke: A Longitudinal Resting-State fMRI Study. Neural Plast 2018;2018:9394156.

18. Li QG, Zhao C, Shan Y, et al. Dynamic Neural Network Changes Revealed by Voxel-Based Functional Connectivity Strength in Left Basal Ganglia Ischemic Stroke. Front Neurosci 2020;14:526645.

19. Whitfield-Gabrieli S, Nieto-Castanon A. Conn: a functional connectivity toolbox for correlated and anticorrelated brain networks. Brain Connect 2012;2:125-41.

20. Lenka A, Bhalsing KS, Panda R, et al. Role of altered cerebello-thalamo-cortical network in the neurobiology of essential tremor. Neuroradiology 2017;59:157-68.

21. Carrera E, Tononi G. Diaschisis: past, present, future. Brain 2014;137:2408-22.

22. Henseler I, Regenbrecht F, Obrig H. Lesion correlates of patholinguistic profiles in chronic aphasia: comparisons of syndrome-, modality- and symptom-level assessment. Brain 2014;137:918-30.

23. Pillay SB, Binder JR, Humphries C, et al. Lesion localization of speech comprehension deficits in chronic aphasia. Neurology 2017;88:970-5.

24. Kim G, Jeong B, Choi M, et al. Neural substrates of subcortical aphasia in subacute stroke: Voxel-based lesion symptom mapping study. J Neurol Sci 2021;420:117266.

25. Balaev V, Petrushevsky A, Martynova O. Changes in Functional Connectivity of Default Mode Network with Auditory and Right Frontoparietal Networks in Poststroke Aphasia. Brain Connect 2016;6:714-23.

26. Harvey DY, Wei T, Ellmore TM, et al. Neuropsychological evidence for the functional role of the uncinate fasciculus in semantic control. Neuropsychologia 2013;51:789-801.

27. Naeser MA, Ho MD, Martin PI, et al. Increased 
Functional Connectivity Within Intrinsic Neural Networks in Chronic Stroke Following Treatment with Red/NearInfrared Transcranial Photobiomodulation: Case Series with Improved Naming in Aphasia. Photobiomodul Photomed Laser Surg 2020;38:115-31.

28. Mirman D, Kraft AE, Harvey DY, et al. Mapping articulatory and grammatical subcomponents of fluency deficits in post-stroke aphasia. Cogn Affect Behav Neurosci 2019;19:1286-98.

29. Sul B, Lee KB, Hong BY, et al. Association of Lesion Location With Long-Term Recovery in Post-stroke Aphasia and Language Deficits. Front Neurol 2019;10:776.

30. Behroozmand R, Phillip L, Johari K, et al. Sensorimotor impairment of speech auditory feedback processing in aphasia. Neuroimage 2018;165:102-11.

31. Buchsbaum BR, Baldo J, Okada K, et al. Conduction aphasia, sensory-motor integration, and phonological short-term memory - an aggregate analysis of lesion and fMRI data. Brain Lang 2011;119:119-28.

32. Kristinsson S, Thors H, Yourganov G, et al. Brain Damage Associated with Impaired Sentence Processing in Acute Aphasia. J Cogn Neurosci 2020;32:256-71.

33. Warren JE, Crinion JT, Lambon Ralph MA, et al. Anterior temporal lobe connectivity correlates with functional outcome after aphasic stroke. Brain 2009; 132:3428-42.

34. Lukic S, Barbieri E, Wang X, et al. Right Hemisphere Grey Matter Volume and Language Functions in Stroke Aphasia. Neural Plast 2017;2017:5601509.

35. Ren CL, Zhang GF, Xia N, et al. Effect of low-frequency rTMS on aphasia in stroke patients: a meta-analysis of randomized controlled trials. PLoS One 2014;9:e102557.

36. Zumbansen A, Black SE, Chen JL, et al. Non-invasive brain stimulation as add-on therapy for subacute poststroke aphasia: a randomized trial (NORTHSTAR). Eur Stroke J 2020;5:402-13.

37. Ren C, Zhang G, Xu X, et al. The Effect of rTMS over the Different Targets on Language Recovery in Stroke Patients with Global Aphasia: A Randomized ShamControlled Study. Biomed Res Int 2019;2019:4589056.

38. Metoki A, Wang Y, Olson IR. The Social Cerebellum: A Large-Scale Investigation of Functional and Structural Specificity and Connectivity. Cereb Cortex 2021. [Epub ahead of print].

39. Van Overwalle F, Manto M, Cattaneo Z, et al. Consensus Paper: Cerebellum and Social Cognition. Cerebellum 2020;19:833-68.
40. Schmahmann JD. Emotional disorders and the cerebellum: Neurobiological substrates, neuropsychiatry, and therapeutic implications. Handb Clin Neurol 2021;183:109-54.

41. Ahmadian $\mathrm{N}$, van Baarsen $\mathrm{K}$, van Zandvoort M, et al. The Cerebellar Cognitive Affective Syndrome-a Meta-analysis. Cerebellum 2019;18:941-50.

42. Mariën P, Ackermann H, Adamaszek M, et al. Consensus paper: Language and the cerebellum: an ongoing enigma. Cerebellum 2014;13:386-410.

43. Pliatsikas C, Johnstone T, Marinis T. Grey matter volume in the cerebellum is related to the processing of grammatical rules in a second language: a structural voxelbased morphometry study. Cerebellum 2014;13:55-63.

44. Sebastian R, Saxena S, Tsapkini K, et al. Cerebellar tDCS: A Novel Approach to Augment Language Treatment Poststroke. Front Hum Neurosci 2016;10:695.

45. Li J, Du D, Gao W, et al. The regional neuronal activity in left posterior middle temporal gyrus is correlated with the severity of chronic aphasia. Neuropsychiatr Dis Treat 2017;13:1937-45.

46. Sebastian R, Long C, Purcell JJ, et al. Imaging network level language recovery after left PCA stroke. Restor Neurol Neurosci 2016;34:473-89.

47. Xu L, Huang L, Cui W, et al. Reorganized functional connectivity of language centers as a possible compensatory mechanism for basal ganglia aphasia. Brain Inj 2020;34:430-7.

48. Visser M, Embleton KV, Jefferies E, et al. The inferior, anterior temporal lobes and semantic memory clarified: novel evidence from distortion-corrected fMRI. Neuropsychologia 2010;48:1689-96.

49. Sakurai Y. Varieties of alexia from fusiform, posterior inferior temporal and posterior occipital gyrus lesions. Behav Neurol 2004;15:35-50.

50. Lin YH, Young IM, Conner AK, et al. Anatomy and White Matter Connections of the Inferior Temporal Gyrus. World Neurosurg 2020;143:e656-66.

51. Price AR, Bonner MF, Peelle JE, et al. Converging evidence for the neuroanatomic basis of combinatorial semantics in the angular gyrus. J Neurosci 2015;35:3276-84.

52. Ramage AE, Aytur S, Ballard KJ. Resting-State Functional Magnetic Resonance Imaging Connectivity Between Semantic and Phonological Regions of Interest May Inform Language Targets in Aphasia. J Speech Lang Hear Res 2020;63:3051-67. 
53. Mårtensson F, Roll M, Lindgren M, et al. Sensory-specific anomic aphasia following left occipital lesions: data from free oral descriptions of concrete word meanings.

Cite this article as: $\mathrm{Xu} \mathrm{X}$, Ren C, Fang H, Li Z, Chen P, Yang J, Wang T. Exploring the functional connectivity characteristics of brain networks in post-stroke patients with global aphasia: a healthy control based resting-state fMRI study. Ann Palliat Med 2021;10(12):12113-12128. doi: 10.21037/apm-21-2750
Neurocase 2014;20:192-207.

(English Language Editor: B. Draper) 